\title{
Impairment of repetitive impulse conduction in experimentally demyelinated and pressure-injured nerves $^{1,2}$
}

\author{
FLOYD A. DAVIS \\ From the Department of Neurology, Rush-Presbyterian-St. Luke's Medical Center, \\ Chicago, Illinois, U.S.A.
}

SUMMARY Repetitive impulse conduction was studied in segmentally demyelinated peripheral nerves in guinea-pigs with experimental allergic neuritis (EAN) and in pressure-injured frog sciatic nerves. Normal guinea-pig sciatic-peroneal nerves maintained at $37^{\circ} \mathrm{C}$ conducted compound action potentials with only minor amplitude decreases at stimulus frequencies up to $200 / \mathrm{sec}$. In contrast, nerves in EAN guinea-pigs maintained at $37^{\circ} \mathrm{C}$ demonstrated a rapidly progressive decrease in action potential amplitude when stimulated as slowly as $10-25 / \mathrm{sec}$. The decrease is greater the higher the frequency of stimulation. At $100 \mathrm{stimuli} / \mathrm{sec}$ all EAN preparations showed more than a $50 \%$ reduction in action potential amplitude. These effects are reversible. In pressure-injured frog sciatic nerves similar effects occurred at stimulus frequencies as low as $50 / \mathrm{sec}$. Normal frog nerves conducted up to 200 impulses/sec with little amplitude decrease. The probable mechanism and clinical significance of these results are discussed.

There is a striking similarity in the aberrations of axonal conduction associated with both peripheral and central nervous system demyelination. Marked conduction slowing as well as blockade have been reported in segmentally demyelinated peripheral nerves associated with experimental allergic neuritis (EAN) (Kaeser and Lambert, 1962; Cragg and Thomas, 1964; Hall, 1967), diphtheritic polyneuritis (McDonald, 1963; Morgan-Hughes, 1968; Lehmann, Tackmann, and Lehmann, 1971), tourniquet paralysis (Mayer and Denny-Brown, 1964), and foreign body reaction (Lehmann and Ule, 1964a; Lehmann and Ule, 1964b). Identical defects have been reported in central nervous system demyelination induced by cerebrospinal fluid barbotage (Mayer, 1966) and by the intraneural injection of diphtheria toxin (McDonald and Sears, 1970). Kaeser and Lambert (1962) have suggested that the decreased conduction velocity in segmentally demyelinated nerve might be related to the loss of myelin, which causes a decrease in the density of action current flowing out through the node of Ranvier and thereby

\footnotetext{
1 Presented, in part, at the Twenty-third Annual Meeting of the American Academy of Neurology, New York, April 1971.

2 Supported by the Morris Multiple Sclerosis Research Fund.
}

prolongs the time needed to trigger an action potential. Similarly, a block of conduction might be due to a reduction of the action current density below the minimum amount needed just to maintain conduction.

More recently, another conduction defect has been observed in both peripheral and central demyelination. An impairment can occur in the propagation of relatively low frequencies of impulses. In segmental demyelination in peripheral nerves, conduction failure has been observed at stimulation frequencies as low as $60 \mathrm{c} / \mathrm{sec}$ (Lehmann, Lehmann, and Tackmann, 1971), while in central nervous system demyelination conduction failure has been observed as low as $290 \mathrm{c} / \mathrm{sec}$ (McDonald and Sears, 1970). These findings might also be related to a reduction in action current density. Such a relationship is suggested by Tasaki's classical studies on conduction in myelinated axons (Tasaki, 1953). When an isolated axon conducts two consecutive impulses such that the second falls within the relative refractory period of the first, the action current generated by the second impulse is decreased; the decrease is greater the closer the second impulse lies in relation to the beginning of the relative refractory period. Though this 
phenomenon causes a slowing of conduction in normal fibres there is no danger of a conduction block (except at very high conduction frequencies - that is, around 900-1000 c/sec) since there is normally a five to seven times excess of action current over the minimal amount just needed to maintain conduction. However, if there is a decrease in this action current excess or so-called 'safety factor', a failure of conduction would be expected to occur at unusually low frequencies. Tasaki has shown that such a phenomenon does occur in lightly narcotized nerve (Tasaki, 1953). He has also demonstrated that the same explanation can account for the historical Wedensky inhibition phenomenon ${ }^{3}$ (Tasaki, 1953).

If Tasaki's ideas apply to the impaired frequency response observed in demyelinated nerve, then this defect cannot be considered to be specific for demyelination. Instead, the same type of conduction defect would be expected to occur in any nerve fibre in which there is a decreased safety factor. For these reasons, segmentally demyelinated peripheral nerves in guinea-pigs with EAN and pressure-injured frog sciatic nerves, both of which have been postulated to have alterations in action current that would result in a decreased safety factor (Lorente de Nó, 1947; Kaeser and Lambert, 1962), have been tested for their response to repetitive electrical stimulation. In both preparations, a similar impairment of frequency response has been observed.

\section{METHODS}

1. In vitro FROG NERVE PREPARATION a. Electrical recording An excised frog (Rana pipiens) sciatic nerve trunk was placed in a stimulating-recording apparatus equipped with paired stainless steel or platinum electrodes. A detailed, illustrated description of the apparatus was presented in a previous publication (Davis and Jacobson, 1971). Electrical stimuli were delivered to the thicker, proximal end of the nerve and compound action potentials were recorded monophasically at the other end by crushing the nerve between the recording electrodes. The preparation was grounded midway between the

\footnotetext{
${ }^{3}$ In 1885 Wedensky reported that a fatigued or narcotized nervemuscle preparation stimulated through its nerve may respond with only a brief initial contraction rather than a sustained tetanus when the stimulation frequency is increased beyond a certain point (Wedensky, 1885). Though this so-called Wedensky inhibition was due to a failure of neuromuscular transmission, it later became clear that the same phenomenon could also occur with localized changes in the nerve. Thus, in a later paper (Wedensky, 1903) he described the same contraction pattern when the nerve was locally narcotized or injured by exposure to high temperatures or concentrated solutions of electrolytes.
}

stimulating and recording electrodes. Stimuli con- $\underline{\underline{O}}$ sisted of $7 \mathrm{sec}$ volleys of $0.1 \mathrm{msec}$ rectangular $z$ electrical pulses at frequencies ranging from $10-\stackrel{\mathbb{}}{\subseteq}$ 250/sec. These were delivered from a Grass S-8 stimulator and stimulus isolation unit. A Grass $\underset{0}{\stackrel{(}{0}}$ constant current unit (CCU-1A) was placed between the stimulus isolation unit and the preparation to offset any effect due to altered electrode resistance. 㝏 The stimulus intensity was set at five times the level

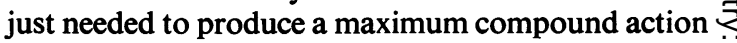
potential. In most experiments a single $0.1 \mathrm{msec}$ rectangular pulse was applied $500 \mathrm{msec}$ after the $\stackrel{5}{+}$ $7 \mathrm{sec}$ volley in order to detect any post-tetanic effects. Compound action potentials were displayed $\frac{\bar{\sigma}}{\bar{D}}$ on a Tektronix dual beam 561-A oscilloscope after $\frac{\bar{\omega}}{\vec{D}}$

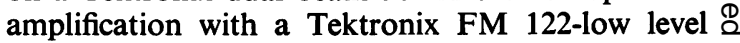
preamplifier and were photographed with a Polaroid is camera.

b. Pressure lesions A short segment of nerve located $\vec{\omega}$ approximately midway between the stimulating and recording electrodes was submerged in a Ringerfilled pool. The temperature of the Ringer solution $\dot{\omega}$ was monitored with a Yellow-Springs model 44 tele- or thermometer and thermistor probe. Segments of the nerve lying outside the pool were carefully encased i⿱⺈ petroleum jelly to prevent drying. Pressure lesions 은 were made in the middle of the segment of nerke submerged in the Ringer-filled pool according to the method of Lorente de Nó (Lorente de Nó, 1947). lesion, $1 \mathrm{~mm}$ long, was made by gently squeezing the nerve with flexible eye forceps; the pressure wos gradually increased until the maximum amplitude of the compound A spike was decreased approximately 60 to $80 \%$. Lorente de Nó has shown that, if this type of lesion is not too wide and the continuity of the nerve fibres is not disrupted, some axons continue to conduct because the safety factor enables impulses to 'jump across' the lesion (Lorente de Nó, $\unrhd$ 1947). His observation that these pressure-injured $\overrightarrow{\vec{P}}$ fibres conduct impulses at a decreased velocity has $\frac{3}{3}$ been confirmed in our previous studies (Davis and $\bar{P}$ Jacobson, 1971). It has also been confirmed that if the length of the lesion is extended beyond 4 or $5 \mathrm{~mm}$ conduction fails completely or nearly so, supporting the idea that the impulse at the site of the $1 \mathrm{~mm}$ lesion 'jumps' rather than 'conducts through' the injured region.

The Ringer solution was adjusted to $\mathrm{pH} \mathbf{7 . 5}$ and had the following composition in millimoles per 0 litre: $\mathrm{NaCl} 115, \mathrm{KCl} 2 \cdot 5, \mathrm{CaCl}_{2} 1 \cdot 8, \mathrm{NaHCO}_{3} 2 \cdot 4$, Tris buffer $1 \cdot 0$.

2. In vivo GUINEA-PIG PREPARATION a. Experi- ๙ু mental allergic neuritis (EAN) EAN was induced N in adult male and female guinea-pigs by the method N of Waksman and Adams (Waksman and Adams, 0 1956). Using aseptic techniques, $2 \mathrm{~g}$ of sciatic nerve from two freshly killed rabbits were cut into short 
lengths and then further sliced with a freezing microtome set at $5 \mu$ advancements. To this tissue mush were added $5 \mathrm{ml}$. isotonic saline, $10 \mathrm{ml}$. Freund's adjuvant (Difco) and $150 \mathrm{mg}$ killed, dried Mycobacterium butyricum (Difco). The mixture was emulsified in a cold electric blender. The resulting emulsion was nearly solid and did not spread when a small drop was added to water. Fifteen guinea pigs weighing between 300 and $500 \mathrm{~g}$ were injected intradermally with $0.1 \mathrm{ml}$. of the emulsion in each forefoot pad. Nine animals developed a moderate to severe clinical picture of EAN; of these, two died before testing. The remaining seven were studied as described next.

b. Electrical recording Under light sodium pentobarbital anaesthesia administered intraperitoneally and supplemented with ether inhalation, the sciatic and peroneal nerves were exposed in the thigh and tibial regions, respectively. The sciatic nərve was severed proximally at the apex of the thigh wound, and all muscles supplied by the sciatic nerve and its branches were denervated. Care was taken to preserve the nerve's blood supply. Skin flaps were raised to enclose a pool of mineral oil that covered the exposed regions of nerve. Mineral oil temperature was maintained at $37^{\circ} \mathrm{C}$ by means of a heat lamp and continuously monitored with a YellowSprings model 44 tele-thermometer and thermistor probe. The proximal portion of the sciatic nerve was stimulated through paired silver electrodes while compound action potentials were recorded with paired platinum electrodes placed along the peroneal nerve. A ground electrode was inserted under the sciatic nerve approximately midway between the stimulating and recording electrodes. Stimulus parameters and recording techniques were the same as those described previously for the frog except for occasional substitution of diphasic recording for monophasic.

c. Histology Some of the normal and EAN guineapig nerves were later fixed for at least one week in $10 \%$ formaldehyde-saline, dehydrated, and embedded in paraffin. Sections were stained with the luxol fast blue-cresyl fast violet method for myelin. Haematoxylin and eosin was also used as a general tissue stain.

\section{RESULTS}

1. GUINEA-PIG Normal guinea-pig sciatic-peroneal nerves maintained at $37^{\circ} \mathrm{C}$ conducted compound action potentials with little or no amplitude variation at stimulation frequencies below 200/sec (Fig. 1, Table 1). In contrast, EAN guinea-pig nerves maintained at $37^{\circ} \mathrm{C}$ demonstrated a decrease in compound action potential amplitude at stimulation frequencies as low as $10-25 / \mathrm{sec}$; this effect was more marked with increasing frequency of stimulation (Fig. 2 and Table 1). The time course of the decline in action potential amplitude varied in different preparations. Some showed a gradual decline as in Fig. 2, while in others the effect was precipitous with the establishment of a steady-like state within seconds. At 100 stimuli per second all EAN preparations demonstrated more than a $50 \%$ decrease in action potential amplitude (Table 1). As seen in Fig. 2, the post-volley

TABLE 1

FREQUENCY RESPONSE IN NORMAL

AND EAN GUINEA-PIG NERVE

\begin{tabular}{|c|c|c|c|c|c|c|c|c|c|}
\hline \multirow[t]{2}{*}{ Exp. } & \multirow[t]{2}{*}{$\begin{array}{l}\text { Temp. } \\
\left({ }^{\circ} \mathrm{C}\right)\end{array}$} & \multicolumn{8}{|c|}{$\begin{array}{l}\text { Percent decrease in compound action potential } \\
\text { amplitude at varying stimulus frequencies }(/ \mathrm{sec})\end{array}$} \\
\hline & & 10 & 25 & 50 & 75 & 100 & 125 & 150 & 200 \\
\hline \multicolumn{10}{|c|}{ I Control } \\
\hline 1 & 37 & & 0 & 0 & 0 & 0 & $2 \cdot 8$ & $2 \cdot 8$ & $2 \cdot 8$ \\
\hline 2 & 37 & & $2 \cdot 6$ & $2 \cdot 6$ & $2 \cdot 5$ & $2 \cdot 5$ & $2 \cdot 5$ & $3 \cdot 7$ & $6 \cdot 2$ \\
\hline 3 & 37 & & 0 & $3 \cdot 8$ & $3 \cdot 8$ & $4 \cdot 0$ & $4 \cdot 0$ & $4 \cdot 0$ & 8.0 \\
\hline 4 & 37 & & 2.9 & 2.9 & 5.9 & $5 \cdot 4$ & $8 \cdot 1$ & 7.9 & $13 \cdot 2$ \\
\hline 5 & 37 & & 0 & $4 \cdot 8$ & $6 \cdot 4$ & $6 \cdot 4$ & $6 \cdot 4$ & $7 \cdot 7$ & $11 \cdot 3$ \\
\hline \multicolumn{10}{|c|}{ II EAN } \\
\hline 1 & 37 & & 16 & 26 & 56 & $69 \cdot 2$ & $67 \cdot 4$ & 69 & \\
\hline 2 & 37 & 6 & 24 & 60 & 74 & 80 & 84 & 86 & \\
\hline 3 & 37 & & $40 \cdot 6$ & 50 & & 56 & & & \\
\hline 4 & 37 & & $16 \cdot 6$ & $31 \cdot 3$ & & & & & \\
\hline 5 & 37.5 & & & & & $80 \cdot 8$ & & & \\
\hline 6 & 37 & & $23 \cdot 1$ & $43 \cdot 3$ & & 63.9 & & & \\
\hline 7 & 37 & 25 & 40 & $62 \cdot 5$ & 85 & 90 & & & \\
\hline
\end{tabular}

stimulus (occuring $0 \cdot 5 \mathrm{sec}$ after cessation of the 7 sec volley) frequently elicited an action potential with a reduced amplitude. This depression is more marked the higher the frequency of the preceding volley (Fig. 2). It is probably related to the post-tetanic depression in EAN reported by Cragg and Thomas (1964). After a $2 \mathrm{~min}$ tetanus at $368 \mathrm{c} / \mathrm{sec}$ they found a depression lasting up to five minutes. Although the time course of this effect was not systematically examined in the present study, it reversed within seconds; in the experiment shown in Fig. 2 the time elapsed between the last two volleys was approximately $45 \mathrm{sec}$. Significant post-tetanic effects were not observed in normal guinea-pig nerves.

Limited histological examinations of some EAN nerves were performed as described in the 


\section{NORMAL G.-Pig $\left(37^{\circ} \mathrm{C}\right)$}

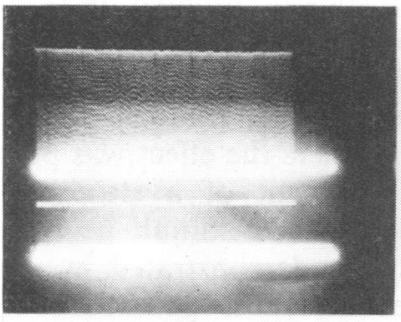

$50 / \mathrm{sec}$

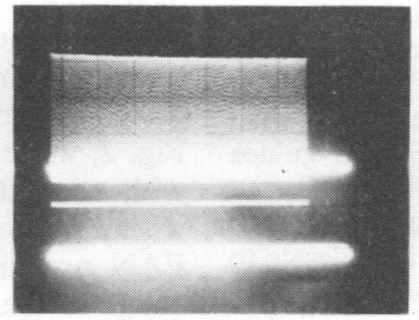

$100 / \mathrm{sec}$

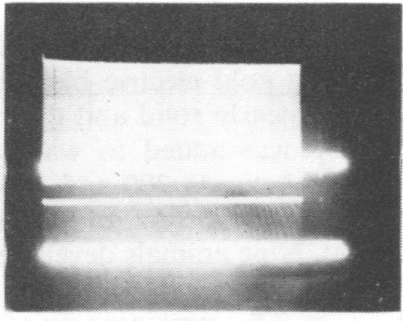

$200 / \mathrm{sec}$

FIG. 1. Successive responses of a normal guinea-pig nerve to $7 \mathrm{sec}$ stimulus volleys at 50 (left), 100 (middle), and 200 (right) c/sec. Stimulus intensity was five times supramaximal. Upper trace: monophasic, compound action potentials. Lower trace: stimulus monitor. Only a relatively small, progressive decline in action potential amplitude occurs even at the highest frequency.

EAN - G.Pig $\left(37^{\circ} \mathrm{C}\right)$

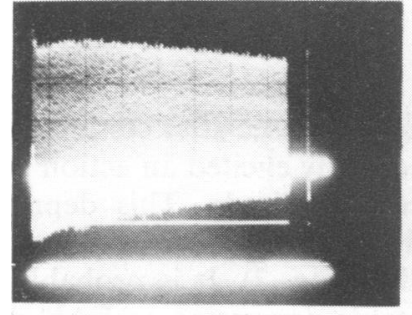

$25 / \sec$

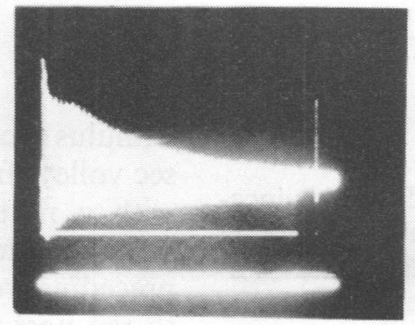

$100 / \mathrm{sec}$

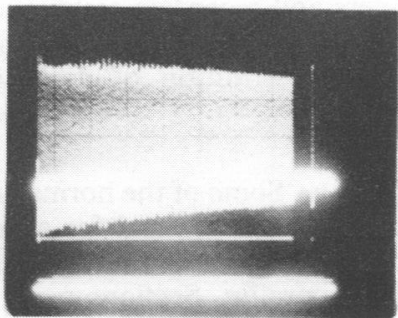

$25 / \sec$

FIG. 2. Successive responses of an EAN guinea-pig nerve to $7 \mathrm{sec}$ stimulus volleys at 25 (left), 100 (middle), and 25 (right) c/sec. Stimulus intensity was five times supramaximal. Upper trace: diphasic compound action potentials. Lower trace: stimulus monitor. A moderate and severe, progressive decline in action potential amplitude occurs at 25 and $100 \mathrm{c} / \mathrm{sec}$, respectively. Note that the single test stimulus occurring $0.5 \mathrm{sec}$ after the termination of the $100 \mathrm{c} / \mathrm{sec}$ volley evokes a submaximal amplitude action potential; approximately 45 sec later the response to $25 \mathrm{c} / \mathrm{sec}$ stimulation indicates that recovery has occurred (compare left and right). 
Methods section. Segments of nerve lying approximately midway between the stimulating and recording electrodes were studied; these showed segmental demyelinating changes identical to those described and illustrated in an earlier study from this laboratory (Davis and Jacobson, 1971).

2. FROG Three separate experimental procedures were employed on each frog sciatic nerve studied. Before making a pressure lesion the nerve was stimulated at varying frequencies using two different stimulus intensities. First the nerve was tested at the usual intensity, which (as described in the Methods section) was five times the minimum amount needed to produce a maximal compound action potential. Nerves stimulated in this manner at frequencies below $200 / \mathrm{sec}$ conducted compound action potentials with little or no amplitude variation (Fig. 3, left; and Table 2, control, A).

Next the stimulus intensity was decreased to a level that produced a submaximal compound action potential; this submaximal stimulus was carefully adjusted so that a slight further decrement in its intensity produced a marked decrease in the action potential amplitude. In
TABLE 2

FREQUENCY RESPONSE IN NORMAL AND PRESSURE-INJURED FROG NERVE

\begin{tabular}{lccccc}
\hline Exp. & $\begin{array}{c}\text { Temp. } \\
\left({ }^{\circ} \mathrm{C}\right)\end{array}$ & $\begin{array}{c}\text { Percent decrease in compound } \\
\text { action potential amplitude at } \\
\text { varying stimulus frequencies }(\mathrm{c} / \mathrm{sec})\end{array}$ \\
\cline { 2 - 4 } & 50 & 100 & 150 & 200 \\
\hline
\end{tabular}

I Control

A. Supramaximal

stimulus

$\begin{array}{llllrr}1 & 23 & 0 & 0 & 4 \cdot 5 & 14 \cdot 3 \\ 2 & 23 & 2 \cdot 4 & 2 \cdot 4 & 7 \cdot 5 & 13 \cdot 1 \\ 3 & 26 \cdot 2 & 0 & 0 & 2 \cdot 4 & 2 \cdot 6 \\ 4 & 28 & 0 \cdot 9 & 2 \cdot 8 & 4 \cdot 6 & 6 \cdot 5 \\ 5 & 26 & 0 & 2 \cdot 0 & 2 \cdot 1 & 8 \cdot 3\end{array}$

B. Submaximal stimulus

$\begin{array}{llllll}1 & 23 & 28 \cdot 1 & 56 \cdot 3 & 71 & 74 \cdot 2 \\ 2 & 23 & 28 \cdot 6 & 50 & 61 \cdot 5 & 82 \cdot 6 \\ 3 & 26 \cdot 2 & 30 \cdot 4 & 40 & 52 \cdot 5 & 60 \\ 4 & 28 & 28 \cdot 1 & 45 \cdot 2 & 59 \cdot 5 & 68 \cdot 7 \\ 5 & 26 & 26 \cdot 7 & 41 \cdot 4 & 50 & 63 \cdot 6\end{array}$

\begin{tabular}{|c|c|c|c|c|c|}
\hline \multicolumn{6}{|c|}{$\begin{array}{l}\text { II Pressure lesion } \\
\text { A. Supramaximal } \\
\text { stimulus }\end{array}$} \\
\hline $\begin{array}{l}1 \\
2 \\
3 \\
4 \\
5\end{array}$ & $\begin{array}{l}23 \\
23 \\
26 \cdot 2 \\
28 \\
26\end{array}$ & $\begin{array}{r}17 \cdot 1 \\
16 \cdot 1 \\
5 \cdot 7 \\
6 \cdot 2 \\
25 \cdot 7\end{array}$ & $\begin{array}{l}34 \cdot 5 \\
28 \cdot 5 \\
25 \cdot 7 \\
16 \cdot 6 \\
48 \cdot 2\end{array}$ & $\begin{array}{l}56.5 \\
53.6 \\
43.4 \\
29.6 \\
58.4\end{array}$ & $\begin{array}{l}70 \cdot 8 \\
65 \cdot 5 \\
53 \cdot 8 \\
36 \\
70\end{array}$ \\
\hline
\end{tabular}

FIG. 3. Responses of a frog nerve to $7 \mathrm{sec}$ stimulus volleys at $100 \mathrm{c} / \mathrm{sec}$ before (left, middle) and after (right) making a pressure-injury. Upper trace: monophasic, compound action potentials. Lower trace: stimulus monitor. Left and middle, response of normal nerve to five times supramaximal and submaximal stimulus intensities, respectively. The submaximal stimulus results in an abrupt and progressive decline in action potential amplitude. Right, after the production of a pressure-injury there is an abrupt and progressive decline in action potential amplitude even with a five times supramaximal stimulus. See Results section for explanation. 
this situation the stimulus can be considered to be just threshold for many fibres. This threshold electrical stimulus is analogous to the amount of action current available for impulse propagation in a nerve fibre whose safety factor is drastically reduced to a value of 1 . As seen in Table 2 (control, B) and Fig. 3 (centre) the same nerves now show a marked decrease in action potential amplitude, which becomes more marked with increasing frequency of stimulation. In Fig. 3 (centre) the initial response decreases precipitously and within several seconds approaches a steady state.

Pressure lesions were then made in the same nerves and the response to five times supramaximal stimulation observed. As seen in Table 2 (pressure lesion) and Fig. 3 (right), the responses are similar to submaximally stimulated normal nerve, with impaired conduction occurring at frequencies as low as $50 / \mathrm{sec}$. In the case of submaximally stimulated nerve the effect is no doubt due to the failure of the threshold stimulus to stimulate during the relative refractory period of the preceding impulse. In the pressure-injured nerve stimulated with a five times supramaximal stimulus the effect is presumed to be related to a decrease in the density of action current available for maintenance of propagation at the site of the lesion. In both instances the offered explanations are analogous, the main difference being that in the former the 'weak link' is the externally applied electrical stimulus, while in the latter it is the intrinsic, natural stimulus (action current).

Unlike the findings with EAN, the pressureinjured frog nerves did not demonstrate a posttetanic depression.

\section{DISCUSSION}

In both the segmentally demyelinated and pressure-injured nerves the demonstrated impairment to repetitive stimulation is similar. There is an abrupt decrease in the amplitude of the compound action potential often approaching a steady-like state within seconds. This decrease can be caused by any one or combination of the following factors. These include a block of conduction in some fibres, a decreased conduction velocity causing increased temporal dispersion or a decrease in the action potential amplitude of individual axons. It is likely that all three factors contribute to the decline in the compound action potential. As mentioned in the introduction, both a slowing of conduction and a decrease in action current (and also action $\underline{\bar{c}}$ potential) occur when an impulse is initiated during the relative refractory period of a pre- $\stackrel{\mathbb{T}}{=}$ ceding impulse. The most severe conduction of defect related to the latter change would be $a \stackrel{\leftarrow}{\frac{c}{6}}$ complete block when the action current falls 0 below the minimum amount needed to maintain conduction. The very marked decreases in com- $\frac{\overline{0}}{\overline{0}}$ pound action potential amplitude observed in $\vec{\gtrless}$ this study are compatible with a conduction: block in some fibres. A definitive answer would, $\stackrel{\vec{\rho}}{\stackrel{9}{+}}$ of course, require a similar study on single? axons.

The abrupt decrease in action potential $\frac{\bar{\omega}}{\widetilde{\sigma}}$ amplitude seen at the beginning of a stimulus $\varrho$ volley is probably an example of Wedensky inhibition. Fibres with severely decreased safety $\overrightarrow{0}$ factors might be able to conduct a limited $\overrightarrow{\vec{\omega}}$ number of closely spaced impulses but with $\stackrel{\omega}{\sigma}$ repetition there would be a gradual whittling down of the action current (if impulses are? separated by intervals less than the relativec refractory period) and an abrupt block woula is eventually occur. The time course of this Wedensky-like inhibition would largely deperfic upon the number and severity of diseased injured axons. If the conduction safety factor ratio was only slightly greater than 1 in a vegु응 large number of fibres, a very large and abru decrease in action potential amplitude would expected. On the other hand, a more widely? distributed range of decreased safety factors in $\mathrm{O}$ the same number of fibres should cause a more gradual decrease in amplitude of the compound action potential. These effects are not prominent $\stackrel{0}{\circ}$ in normal nerves since the safety factor ratio is around 5 to 7 (Tasaki, 1953), allowing conduc- $\overline{0}$ tion to continue even when impulses fall in the relative refractory period of preceding activity. In fact, in normal nerves, the least interval between successive impulses is only slightly greater than the absolute refractory period: (Tasaki, 1953).

The similarity of the responses in segmentally demyelinated nerve and pressure-injured nerve $₹$ support the idea that any adverse condition 을 which tends to depress the conduction safety factor will result in an impaired response to을 repetitive stimulation. As mentioned in the N introduction both segmentally demyelinated and pressure-injured nerves have been postulated to $N$ have a decreased safety factor. It is important to stress, however, that the decreased safety factors are brought about by different means in these $\frac{0}{\Phi}$ 
two preparations. In EAN the action current density is believed to be decreased by the widened nodal gap as well as by shunting of current through demyelinated internodes (Kaeser and Lambert, 1962). In pressure-injured nerve where the impulse is believed to 'jump over' the blocked segment, current must flow through a longer segment and therefore through a greater internal longitudinal resistance. In addition, the current presumably still flowing out of the nodes in the blocked segment is unavailable for propagation. It is not known whether or not alterations in excitability also contribute to safety factor changes in these preparations. A discussion of conduction parameters that can affect the safety factor has been presented elsewhere (Davis, 1970).

Phenomenologically, the similarity of conduction defects in segmental demyelination and pressure-injured nerve includes more than the frequency response changes. Both preparations exhibit slowing of conduction (Lorente de Nó, 1947; Kaeser and Lambert, 1962), conduction block (Lorente de Nó, 1947; Cragg and Thomas, 1964) and an increased sensitivity to temperature (Davis and Jacobson, 1971). The usefulness of the pressure-injured preparation in studying possible conduction defects in demyelinating diseases such as multiple sclerosis has been described in a previous communication (Davis and Jacobson, 1971). It has been shown that conduction in pressure-injured nerves is improved by lowering the concentration of calcium ions around the site of the lesion. This effect is believed to be due to a decrease in threshold caused by the calcium depletion which, in turn, increases the conduction safety factor. This idea was tested clinically in multiple sclerosis patients by observing the effects of hypocalcaemic inducing agents on visual and oculomotor function. Transient improvement in scotomas, nystagmus, and oculomotor paresis occurred with intravenous infusions of sodium bicarbonate or disodium edetate $\left(\mathrm{Na}_{2} \mathrm{EDTA}\right)$ (Davis, Becker, Michael, and Sorensen, 1970).

The demonstration that conduction failure can occur at very low frequencies in segmentally demyelinated nerve raises the possibility that the phenomenon may have clinical significance in the segmental demyelinating neuropathies as well as in central nervous system demyelinating disorders. McDonald and Sears (1970) have suggested that the failure of demyelinated axons faithfully to conduct repetitive activity could help explain such symptoms as the rapid increase of muscle weakness during exercise. The Wedensky-like inhibition could also explain certain sensory phenomena. For example, multiple sclerosis patients who have optic nerve lesions can experience a 'fading out' of vision while looking at an object continuously for several seconds (Bender, 1963). This adaptation phenomenon is short-lived and can usually be reversed simply by closing the eyes for several seconds.

It would appear that studies on conduction defects in experimental demyelinated models and even pressure-injured nerve can contribute to our understanding of the pathophysiology of clinical demyelinating conditions. This appears to be particularly true when phenomena are viewed in terms of general concepts of axonal physiology; with closer probing of specific conduction parameters such as resting potential, threshold, action current, spike amplitudeduration, etc., it is likely that important dissimilarities will emerge.

The author wishes to thank Dr. Raymond Clasen for preparing histological sections and Dr. Samuel Jacobson for technical assistance.

\section{REFERENCES}

Bender, M. B. (1963). Disorders in visual perception. In Problems of Dynamic Neurology: An International Volume, pp. 319-375. Edited by L. Halpern. Rothschild Hadassah Hospital: Jerusalem.

Cragg, B. G., and Thomas, P. K. (1964). Changes in nerve conduction in experimental allergic neuritis. Journal of Neurology, Neurosurgery, and Psychiatry, 27, 106-115.

Davis, F. A. (1970). Pathophysiology of multiple sclerosis and related clinical implications. In Modern Treatment, 7, 890-902. Edited by G. M. Lehrer. Harper and Row: New York.

Davis, F. A., Becker, F. O., Michael, J. A., and Sorensen, E. (1970). Effect of intravenous sodium bicarbonate, disodium edetate $\left(\mathrm{Na}_{2} \mathrm{EDTA}\right)$, and hyperventilation on visual and oculomotor signs in multiple sclerosis. Journal of Neurology, Neurosurgery, and Psychiatry, 33, 723-732.

Davis, F. A., and Jacobson, S. (1971). Altered thermal sensitivity in injured and demyelinated nerve. A possible model of temperature effects in multiple sclerosis. Journal of Neurology, Neurosurgery, and Psychiatry, 34, 551-561.

Hall, J. I. (1967). Studies on demyelinated peripheral nerves in guinea-pigs with experimental allergic neuritis: a histological and electrophysiological study. II, Electrophysiological observations. Brain, 90, 313-332.

Kaeser, H. E., and Lambert, E. H. (1962). Nerve function studies in experimental polyneuritis. Electroencephalography and Clinical Neurophysiology, Suppl. 22, 29-35.

Lehmann, H. J., Lehmann, G., and Tackmann, W. (1971). Refraktärperiode und Übermittlung von Serienimpulsen im N. tibialis des Meerschweinchens bei experimenteller allergischer Neuritis. Neurologische Zentralblatt, 199, 67-85.

Lehmann, H. J., Tackmann, W., and Lehmann, G. (1971). Funktionsänderung markhaltiger Nervenfasern im N. 
tibialis des Meerschweinchens bei postdiphtherischer Polyneuritis. Neurologische Zentralblatt, 199, 86-104.

Lehmann, H. J., and Ule, G. (1964a). Ultrastructure and conduction velocity in demyelinated nerve fibres. (Abstract.) International Meeting on Electromyography, Copenhagen, 1963. Electroencephalography and Clinical Neurophysiology, 17, 98.

Lehmann, H. J., and Ule, G. (1964b). Electrophysiological findings and structural changes in circumscript inflammation of peripheral nerves. Progress in Brain Research, 6, 169-173.

Lorente de Nó, R. (1947). A study of nerve physiology. Studies from the Rockefeller Institute for Medical Research, 131, Pt. 1.

McDonald, W. I. (1963). The effects of experimental demyelination on conduction in peripheral nerve: a histological and electrophysiological study. II. Electrophysiological observations. Brain, 86, 501-524.

McDonald, W. I., and Sears, T. A. (1970). The effects of experimental demyelination on conduction in the central nervous system. Brain, 93, 583-598.
Mayer, R. F. (1966). Conduction velocity in spinal cord during experimental demyelination in the cat. Transactions of the American Neurological Association, 91, 294-296.

Mayer, R. F., and Denny-Brown, D. (1964). Conduction velocity in peripheral nerve during experimental demyelination in the cat. Neurology (Minneap.), 14, 714-726.

Morgan-Hughes, J. A. (1968). Experimental diphtheritic $\underset{\varrho}{0}$ neuropathy: a pathological and electrophysiological study. Journal of the Neurological Sciences, 7, 157-175.

Tasaki, I. (1953). Nervous Transmission. Thomas: Springfield, Ill.

Waksman, B. H., and Adams, R. D. (1956). A comparative study of experimental allergic neuritis in the rabbit, guinea pig, and mouse. Journal of Neuropathology and Experimental Neurology, 15, 293-333.

Wedensky, N. E. (1885). Ueber einige Beziehungen zwischen der Reizstärke und der Tetanushöhe bei indirecter Reizung. Pflügers Archiv für die gesamte Physiologie des Menschen und der Tiere, 37, 69-72.

Wedensky, N. E. (1903). Die Erregung, Hemmung und $\mathbb{D}$ Narkose. Pflügers Archiv für die gesamte Physiologie des Menschen und der Tiere, 100, 1-144. 